\title{
Predicting Data-Dependent Jitter
}

James Buckwalter, Student Member, IEEE, Behnam Analui, Student Member, IEEE, and Ali Hajimiri, Member, IEEE

\begin{abstract}
An analysis for calculating data-dependent jitter (DDJ) in a first-order system is introduced. The predicted DDJ features unique threshold crossing times with self-similar geometry. An approximation for DDJ in second-order systems is described in terms of the damping factor and natural frequency. Higher order responses demonstrate conditions under which unique threshold crossing times do not exist and total jitter is minimized. The DDJ predictions are verified with jitter measurements in a bandwidth-limited amplifier. The predictions for both firstand second-order systems anticipate the features of the observed jitter.
\end{abstract}

Index Terms-Data-dependent jitter (DDJ), intersymbol interference, jitter.

\section{INTRODUCTION}

A $S$ BIT RATES increase, timing accuracy becomes more critical to system performance. In a transmitter, any deviation from the reference clock translates to timing jitter in the transmitted electrical or optical signal. The data sequence timing is further corrupted during transmission over a dispersive channel. Nonidealities of the receiver, e.g., asymmetric rise and fall times and sampling point variations, exacerbate the overall timing jitter and disturb the data regeneration and clock recovery, resulting in an increased bit-error rate. Consequently, high-speed serial links rely on careful jitter analysis [1].

Timing jitter is composed of random jitter (RJ) and deterministic jitter (DJ). RJ is related to voltage noise through the slope of the signal [2] and the phase noise of the transmitter clock. DJ is timing deviations arising from nonidealities such as limited bandwidth, signal reflection, duty-cycle distortion, or power-supply noise [1], [3]. Depending on the noise source, DJ is classified into categories. Data-dependent jitter (DDJ) is a prominent form of DJ caused by the previous transmitted data symbols.

Although measurement and characterization techniques of RJ and DJ exist in the literature [3]-[5], the analysis of the relationship of DDJ to system parameters is often overlooked. Limited bandwidth of the transmitter or receiver blocks (e.g., modulator driver, transimpedance amplifier), or electromagnetic reflections from poorly matched interfaces cause DDJ. Relating DDJ to system response and the bit rate provides insight to managing the communication link jitter budget and designing circuits to minimize jitter.

Manuscript received January 12, 2004; revised March 10, 2004. This work was supported by the Lee Center for Advanced Networking. This paper was recommended by Associate Editor C.-W. Wu.

The authors are with the California Institute of Technology, Pasadena, CA 91125 USA (e-mail: jamesb@caltech.edu; behnam@caltech.edu; hajimiri@caltech.edu).

Digital Object Identifier 10.1109/TCSII.2004.834537

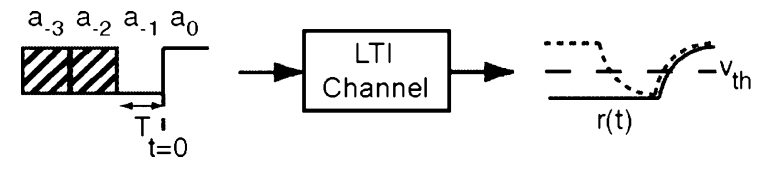

Fig. 1. Impact of a communication channel modeled with LTI system on the timing deviation of the data signal.

We present a theoretical study on DDJ. The analysis provides equations for DDJ that predict the timing impairment of the data in first-and second-order systems. We compare these predictions with measurements taken with a broadband amplifier and bandwidth-limiting output filters. The predictions provide intuition about tailoring the system response to improve the DDJ.

\section{DDJ}

The response of a causal system with finite bandwidth to a data bit is not only determined by the current bit but also the previous bits. Effectively, the system response has limited bandwidth and retains memory of the previous bits. At each transition, the sequence of previous bits shifts the output amplitude and changes the time the signal crosses a decision threshold $v_{\text {th }}$. This timing deviation, illustrated in Fig. 1 for 3 bits, depends on the particular data sequence.

The system response determines the behavior of DDJ. The transmitted nonreturn-to-zero (NRZ) data signal is

$$
\begin{array}{r}
x(t)=\sum_{n=-\infty}^{0} a_{n} p(t-n T), \\
\text { where } p(t)= \begin{cases}1, & 0<t \leq T \\
0, & t \leq 0 ; \quad T<t .\end{cases}
\end{array}
$$

Here, $a_{n}$ is the binary value, $p(t)$ is the pulse response, and $T$ is the bit period. The received signal is affected by the response of the channel. Typically this is a linear time-invariant (LTI) system and the received pulse response is related to the channel response

$$
r(t)=\sum_{n=-\infty}^{0} a_{n} g(t-n T), \quad \text { where } g(t)=h(t) \otimes p(t)
$$

where $h(t)$ is the channel impulse response and $g(t)$ is the received pulse response. Now, we determine the threshold crossing time $t_{c}$ for arbitrary values of previous bits

$$
v_{\text {th }}=r\left(t_{c}\right)=\sum_{n=-\infty}^{0} a_{n} g\left(t_{c}-n T\right)
$$

where $v_{\text {th }}$ is the voltage threshold. In (3), the threshold crossing time can be solved implicitly considering the function $g(t)$. In 


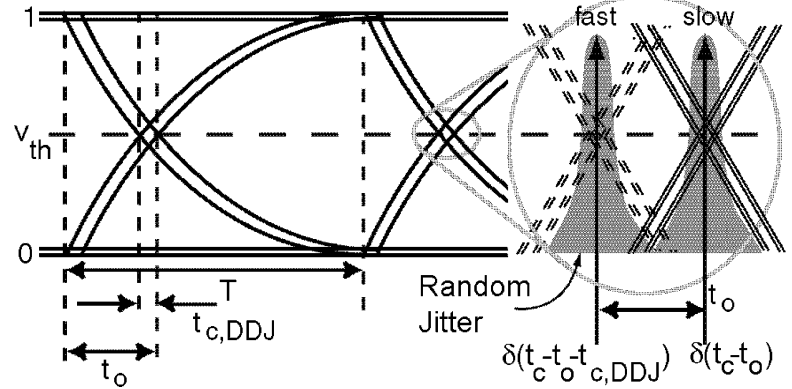

Fig. 2. DDJ apparent in the data eye and the resulting jitter histogram.

the following sections, the response is considered as first- and second order.

DDJ is the deviation of $t_{c}$ for arbitrary bit sequences. While $t_{c}$ is implicit in (3), one solution is the step response. If all previous bits are zero, $g\left(t_{o}\right)=v_{\text {th }}$ where $t_{o}$ is the threshold crossing time for the step response. If two previous bits are considered, there are four sequences with transitions at the current bit: 001, 101, 110 , and 010 . Assuming that $v_{\text {th }}=0.5$, the rising and falling transitions are symmetric and there are two $t_{c}$ for the 001 and 101 sequences.

The jitter histogram shown in Fig. 2 represents the total jitter at the transitions of the data eye. Since RJ and DDJ are independent, the total jitter is the convolution of RJ shown in gray, and DDJ

$$
\operatorname{pdf}_{\text {TotalJitter }}=\operatorname{pdf}_{\mathrm{RJ}} \otimes \operatorname{pdf}_{\mathrm{DDJ}}
$$

RJ is typically a Gaussian distribution and the location of the jitter peaks is found from the DDJ probability distribution. Since NRZ data is generated stochastically, DDJ is a probability density function (pdf) with discrete values of $t_{c}$. For the 001 and 101 sequences, the first-order DDJ pdf consists of a double dirac function as modeled in [1]

$$
\operatorname{pdf}_{\mathrm{DDJ}}\left(t_{c}\right)=\frac{1}{2}\left[\delta\left(t_{c}-t_{o}\right)+\delta\left(t_{c}-t_{o}-t_{c, \mathrm{DDJ}}\right)\right]
$$

If additional bits are considered, each delta function in (5) splits into two delta functions (and, correspondingly, jitter peaks) with half the probability per peak.

Equation (4) indicates that RJ is mapped onto each DDJ peak as illustrated in Fig. 2. The magnitude of the total jitter increases with the separation between the delta functions described in (5). Therefore, this is an important figure of merit for deterministic and total jitter [1], [3]. Using (3) and a model for the step response, an accurate expression for DDJ is found and the jitter peaks (4) of are predicted.

\section{FIRST-ORDER RESPONSE}

Reducing the DDJ of the entire communication link into the contributions of individual blocks allows jitter analysis of each component. Oftentimes, the blocks can be approximated with a

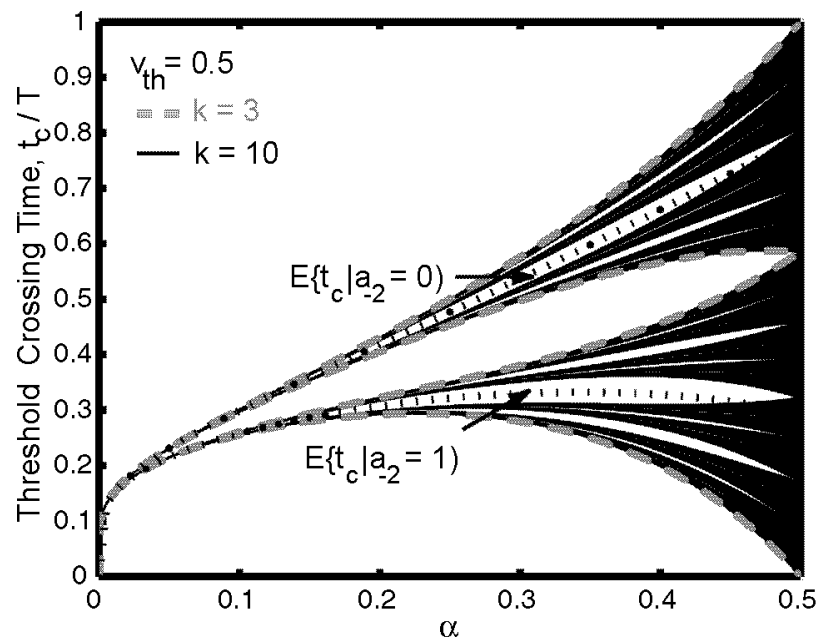

Fig. 3. Normalized threshold crossing time with respect to the bit rate and system bandwidth for the first-order system. The dotted lines are the means of the fast and slow sets of data sequences.

first-order system. In this case, the received pulse response can be written analytically

$$
g(t)=\left\{\begin{array}{cc}
0, & t<0 \\
1-e^{-\frac{t}{\tau}}, & 0<t<T \\
e^{-\frac{(t-T)}{\tau}}\left(1-e^{-\frac{T}{\tau}}\right), & T<t .
\end{array}\right.
$$

Substituting (6) into (3), we discard noncausal terms

$$
v_{\text {th }}=a_{0}\left(1-e^{-\frac{t_{c}}{\tau}}\right)+\sum_{n=-\infty}^{-1} a_{n} e^{-\frac{t_{c}}{\tau}}\left[e^{\frac{(n+1) T}{\tau}}-e^{\frac{n T}{\tau}}\right]
$$

We define $\alpha \equiv e^{-T / \tau}$, which represents the ratio between the bandwidth and the bit rate of the system. This relationship for $\alpha$ magnifies the ratio. For instance, if $\mathrm{BW}=0.7 / T, \alpha=$ 0.0123 . But if $\mathrm{BW}=0.35 / T, \alpha=0.111$. Since $t_{c}$ can be extracted from the summation, (7) has a closed-form solution for the first-order response

$$
t_{c}=\tau \cdot \ln \left[\frac{-a_{0}+\sum_{n=-\infty}^{-1} a_{n}\left[\alpha^{-(n+1)}-\alpha^{-n}\right]}{v_{\mathrm{th}}-a_{0}}\right] .
$$

Equation (8) indicates how each prior bit affects $t_{c}$. Because $\alpha$ is less than one, it is clear that $\alpha^{k}$ is exponentially decreasing. Therefore, $a_{-2}$ has the most dominant effect on the DDJ and the previous bits have less impact on the current threshold crossing time.

If we consider a sequence of $k$ bits, there are $2^{k-1}$ sequences with data transitions at the current bit. Therefore, each sequence is mapped to a particular $t_{c}$ from (8). Each line in Fig. 3 corresponds to one of the $2^{k-1}$ data sequences. As $\alpha$ decreases (the bandwidth of the system increases), the values of $t_{c}$ converge and the deviation of $t_{c}$ is small. However, reducing the bandwidth splits the $t_{c}$ into fast and slow groups. The slow response is associated with the 001 sequence and the fast response is the result of the 101 sequence because the reduced bandwidth prevents the signal from completely reaching each binary level. These terms then split into additional fast and slow groups. If 
the RJ is small enough, the splitting of the jitter peaks into slow and fast groups is resolvable.

The $t_{c}$ values associated with the data sequences can be classified by the fast and slow sets. The slow set is denoted by $t_{c \mid 0}$ for $a_{-2}=0$ and $t_{c \mid 1}$ denotes the fast set for $a_{-2}=1$. The peaks of the DDJ pdf in (5) are represented by $t_{c \mid 0}$ and $t_{c \mid 1}$, the two separate peaks with equal heights in Fig. 2. Therefore, the first-order DDJ can be equivalently defined as the difference between the means conditioned on the penultimate bit

$$
t_{c, \mathrm{DDJ}}=E\left\{t_{c \mid 0}\right\}-E\left\{t_{c \mid 1}\right\} .
$$

For equiprobable NRZ data, each mean can be found from (8) by substituting in the possible data sequences. Assuming $v_{\text {th }}=$ 0.5 , the difference is

$$
t_{c, \mathrm{DDJ}}=\frac{\tau}{2} \ln \frac{1+\alpha}{1-\alpha+\alpha^{2}} .
$$

Equation (10) directly relates DDJ to the system bandwidth and bit rate. For greater $\alpha$, i.e., smaller ratio of the system bandwidth to the bit rate, the impact of additional bits appears in the jitter histogram as predicting by the splitting and spreading of the $t_{c}$ in Fig. 3. For instance, when $\alpha$ is such that the effects of both $a_{-2}$ and $a_{-3}$ are significant, the jitter histogram will have four distinct peaks instead of two. In this case, the means can be calculated by conditioning on $a_{-3}$. At each $\alpha$, we can magnify any $t_{c}$ locally in Fig. 3 and find the same structure. In general, the jitter histogram of the DDJ is self-similar and forms a fractal.

\section{SECOND-ORDER RESPONSE}

The expression of $g(t)$ for second-order systems is a more complex function of time. In general, (3) will not have a closed form solution because $t_{c}$ cannot be separated from the summation as in (7). However, linearization techniques such as the Taylor series expansion approximate the DDJ for higher order systems [6]. While numerical methods can be introduced, series expansion provides insight about the relationship of the system response to the observed DDJ.

Qualitatively, the possible behavior of second-order systems includes overdamped, critically damped, and underdamped responses. Underdamped systems have complex poles that result in ringing. This resonance impacts the strength of previous bits on the current $t_{c}$. For example, the 001 sequence and 101 sequence were demonstrated for the first-order system to result in the slow and fast $t_{c}$, respectively. However, for a second-order response, these roles can reverse. This implies the existence of parameters that result in minimization of the DDJ.

A first-order Taylor series approximates the step response of the second-order system

$$
g(t-n T)=g\left(t_{o}-n T\right)+\left(t-t_{o}\right) g^{(1)}\left(t_{o}-n T\right) .
$$

The superscript denotes the order of the derivative. Substituting (11) into (3), the threshold crossing time, $t_{c}$, is

$$
t_{c}=t_{o}+\frac{v_{\mathrm{th}}-\sum_{n=-\infty}^{0} a_{n} g\left(t_{o}-n T\right)}{\sum_{n=-\infty}^{0} a_{n} g^{(1)}\left(t_{o}-n T\right)} .
$$

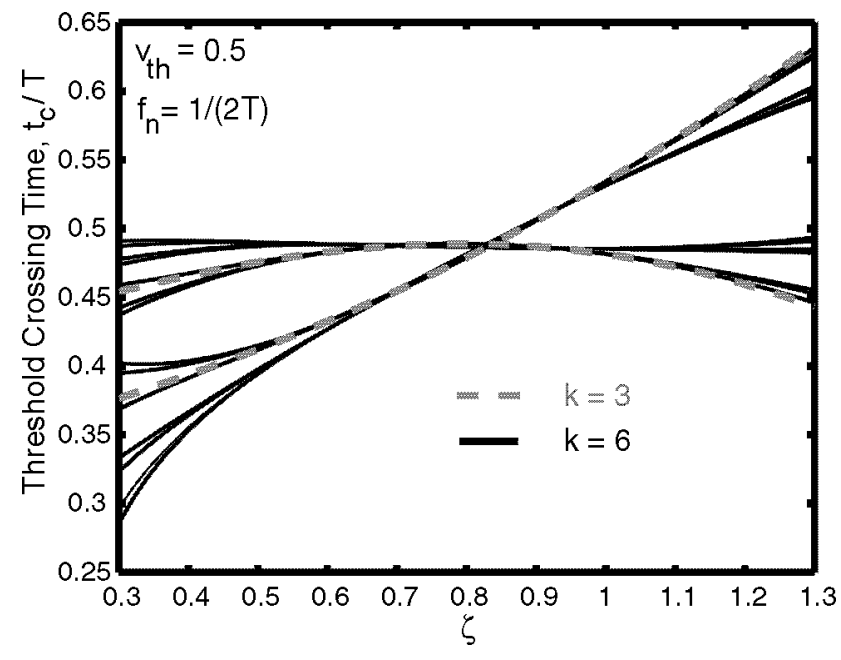

Fig. 4. Threshold crossing time with respect to the bit rate and system bandwidth for second-order system. The intersection of the dashed lines demonstrates the DDJ minimization.

Notably, the denominator contains the slope and the numerator contains bit period delayed values of the pulse response. This relationship suggests that slow waveforms suffer from greater DDJ. Considering $k$-length sequences of bits, the exact solution can be solved numerically. Fig. 4 is the $t_{c}$ as a function of the damping factor at a fixed bandwidth. The 001 and 101 sequences, shown in gray, do indeed intersect for damping factor of about 0.85 . This intersection verifies the anticipated DDJ minimization. Since the RJ is convolved with the DDJ, such system responses demonstrate a local minima for the total jitter.

Equation (12) can be simplified if we consider only three bits and $v_{\text {th }}=0.5$. For 001 and 110 sequences, (12) is $t_{o}$ since, by definition, $g\left(t_{o}\right)=v_{\mathrm{th}}$. For the 101 and 010 sequences

$$
t_{c, \mathrm{DDJ}}=-\frac{v_{\mathrm{th}}-g\left(t_{o}+T\right)}{g^{(1)}\left(t_{o}+T\right)} .
$$

If there is no ringing, the denominator will be positive (for a rising edge). Therefore, the threshhold crossing time deviation described by (13)is positive since $v_{\mathrm{th}}-g\left(t_{o}+T\right)$ is necessarily negative. Alternatively, if there is ringing, the denominator can be negative. The sign of the threshhold crossing time deviation is determined by the sign of the slope and residual pulse response 1-bit period later. Hence, jitter minimization occurs when there is an overshoot such that the response satisfies $g\left(t_{o}+T\right)=v_{\text {th }}$. This observation concurs with the criteria suggested by Gibby and Smith for pulse distortionless transmission [7]. Specifically, the received pulse response should be a function such as illustrated in Fig. 5. At the step threshold crossing time, $t_{o}, g(t)$ reaches the voltage threshold. Two bit periods later, the response should be zero to avoid changing the threshold crossing time of other bits. Finally, (13) can clearly be positive or negative depending on the sign of $v_{\mathrm{th}}-g\left(t_{o}+T\right)$.

\section{EXPERIMENTAL RESULTS}

We demonstrated the accuracy of the jitter expressions with a broadband amplifier and bandwidth-limiting output filter. To measure the jitter resulting from a first-order response, a series 


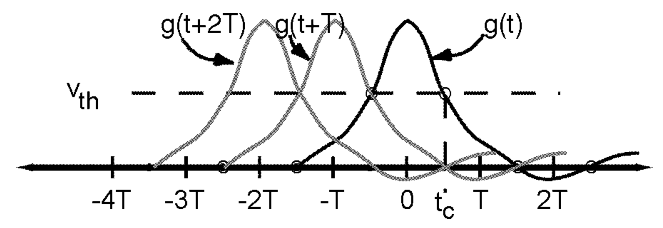

Fig. 5. Hypothetical pulse response that minimizes jitter for second-order system.

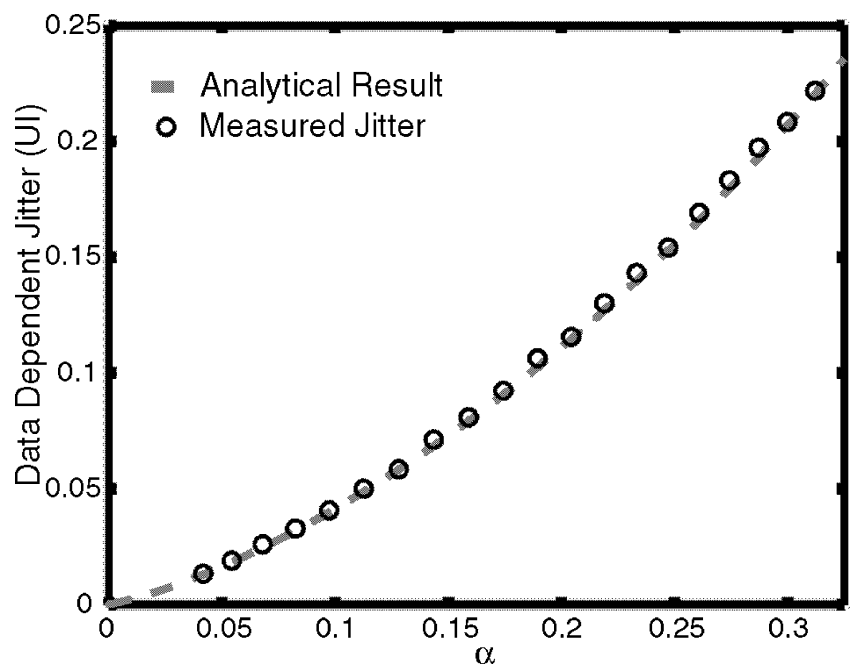

Fig. 6. Comparison of first-order response DDJ prediction with experimental results.

$R C$ filter with bandwidth of $25 \mathrm{MHz}$ was placed between an amplifier and an oscilloscope. The input signal is a $2^{7}-1$ pseudorandom bit sequence (PRBS) and the threshold crossings in the data eye were observed. Since $R C$ filters provide poor matching, the bit rate was scanned from $50 \mathrm{Mb} / \mathrm{s}$ to around $150 \mathrm{Mb} / \mathrm{s}$ to avoid the impact of signal reflection. Furthermore, the low bit rate reduces the amount of RJ and alleviates the problem of resolving the second layer of jitter peaks described in Fig. 3.

The jitter peaks of the jitter histogram were averaged to find the two dominant fast and slow peaks and the relative time between these peaks determined the measured $t_{c, \text { DDJ. Fig. } 6 \text { plots }}$ these measured jitter peaks with the predicted values in (10). Two data eyes are recorded at different $\alpha$ to demonstrate the anticipated peak doubling in Fig. 7. The first data eye corresponds to BW $=0.36 / T$. In Fig. 7(b) each of the jitter peaks has split into a second set of fast and slow jitter peaks. In this case, $\mathrm{BW}=0.18 / T$. Furthermore, additional jitter peaks are clear from the individual traces.

The same experimental setup was used to verify the DDJ for a second-order response. An $L C$ filter with natural frequency of $75 \mathrm{MHz}$ and damping factor of 0.7 was placed between the amplifier and the oscilloscope and the bit rate was scanned. Fig. 8 demonstrates good agreement between the predicted and measured DDJ. Near the slow and fast response intersection, the DDJ is undetectable and these points have been neglected. However, the prediction seems to be accurate with the actual DDJ minimum. The negative values of DDJ do not mean that the jitter is negative but that the relative positions of $E\left\{t_{c \mid 0}\right\}$ and $E\left\{t_{c \mid 1}\right\}$ switch. Therefore, the total jitter is always nonnegative, but the negative sign stresses the dynamics of the response. The
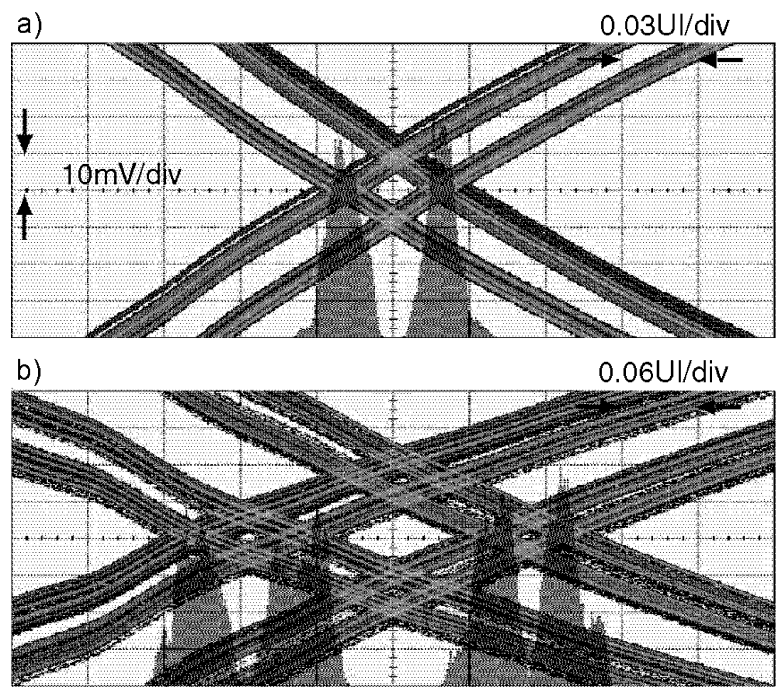

Fig. 7. Data eyes for first-order response at (a) $\alpha=0.1$ and (b) $\alpha=0.31$.

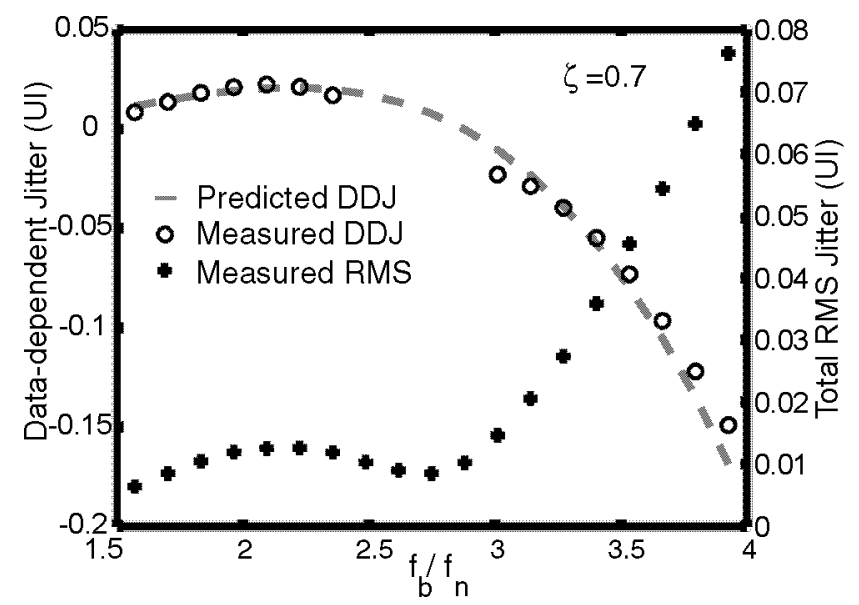

Fig. 8. Comparison of second-order prediction and measured DDJ on the left axis and corresponding RMS jitter on the right axis.
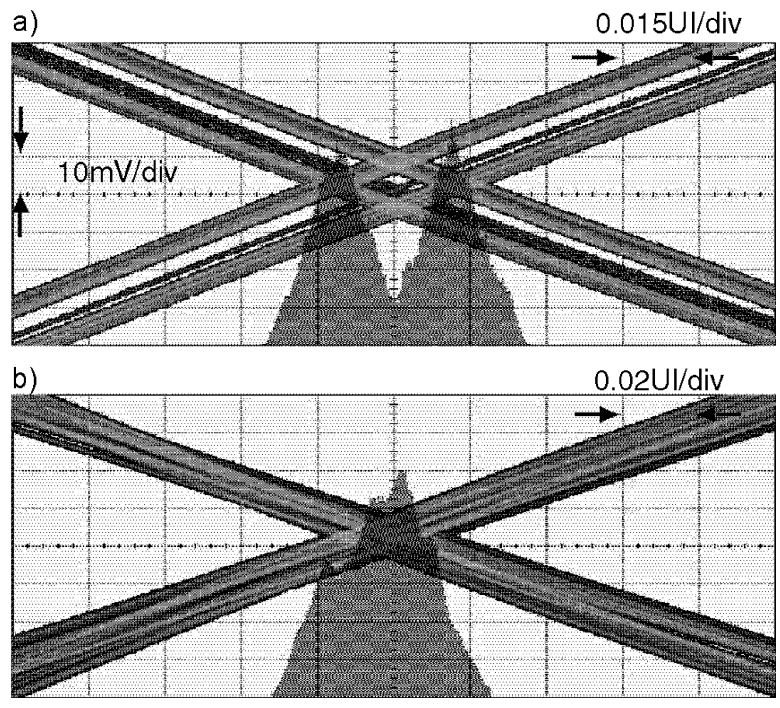

Fig. 9. Threshold crossing eye diagram with superimposed histogram at normalized bit rate of (a) 2 and (b) 2.9 as determined from Fig. 8 .

zero crossing of the curve corresponding to the minimum DDJ is also a local minimum of the total jitter as illustrated on the 


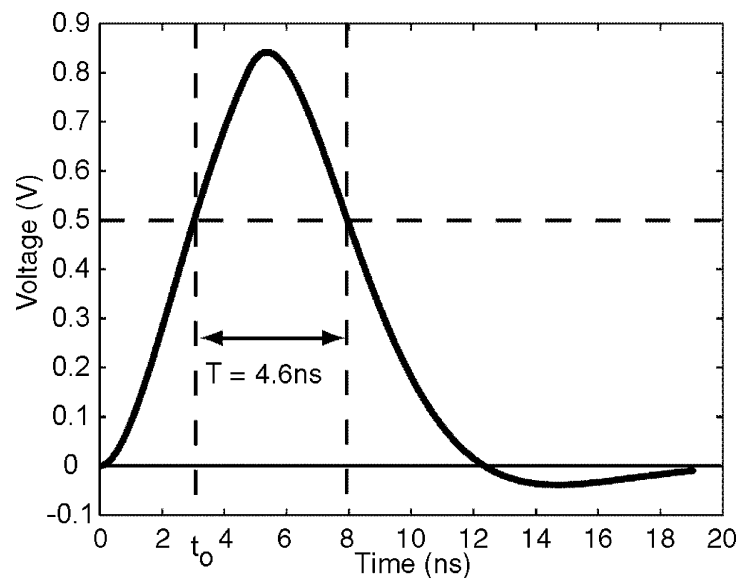

Fig. 10. Received pulse response at the point of jitter minimization and the calculated waveform

second axis in Fig. 8. This minimum provides guideline to design low bandwidth blocks and still achieve low timing jitter. It is notable that the bit rate must be cut by nearly $40 \%$ to reach the same RMS jitter. Fig. 9 demonstrates the transitions of the data eye diagrams at two different bit rates. The first eye diagram is at the normalized bit rate of 2 , before the DDJ minimum. The second eye diagram is at 2.9 near the DDJ minimum. Clearly, the superimposed jitter histogram reflects the lower RMS jitter at the higher bit rate.

Finally, the pulse response is plotted to see how the jitter minimization agrees with the prediction in Fig. 10. The second-order filter with the given parameters was simulated with a 010 sequence. To satisfy $g\left(t_{o}+T\right)=v_{\mathrm{th}}$, we have determined that $T=4.6$ ns. Clearly, this indicates that the bit rate that satisfies the zero jitter condition is $218 \mathrm{Mb} / \mathrm{s}$. From Fig. 8, this point is $2.8 f_{n}=210 \mathrm{Mb} / \mathrm{s}$. Therefore, we have anticipated from the pulse response with good accuracy the zero jitter condition.

\section{CONCLUSION}

This paper develops an analysis of DDJ and provides closed-form solutions for first-order responses. A first-order
Taylor series is used to analyze the general higher order response in terms of the step response. This series is applied specifically to a second-order response.

The first-order response results in jitter peaks that split into fast and slow groups as the bandwidth is reduced. The secondorder response results in jitter peaks that intersect and the fast and slow responses have the same threshold crossing time. This is particularly important because the RJ is convolved around the peaks of the DJ and minimizing the DDJ also minimizes the total jitter.

A broadband amplifier is tested with a first-and second-order filter to verify the predicted locations of the jitter peaks. The predicted and measured values of the DDJ are reasonably close over a broad range of bit rates. Furthermore, the data eyes are provided to illustrate the jitter histogram and DDJ that occurs under these conditions.

\section{ACKNOWLEDGMENT}

The authors would like to thank members of the Caltech High-Speed Integrated Circuit (CHIC) group for their suggestions, and the reviewers for their comments and attention.

\section{REFERENCES}

[1] International Committee for Information Technology Standardization (INCITS), "Fiber channel-Methodologies for jitter and signal quality specification-MJSQ," INCITS, Tech. Rep., Mar. 10, 2003.

[2] A. A. Abidi and R. G. Meyer, "Noise in relaxation oscillators," IEEE J. Solid-State Circuits, vol. SC-18, pp. 794-802, June 1983.

[3] Y. Takasaki, Digital Transmission Design and Jitter Analysis. Boston, MA: Artech House, 1991.

[4] Y. Cai, S. A. Werner, G. J. Zhang, M. J. Olsen, and R. D. Brink, "Jitter testing for multi-gigabit backplane SerDes-techniques to decompose and combine various types of jitter," in Proc. IEEE Int. Test Conf., 2002, pp. 700-708

[5] M. Shimanouchi, "An approach to consistent jitter modeling for various aspects and measurement methods," in Proc. IEEE Int. Test Conf., 2001, pp. 700-709.

[6] B. R. Saltzberg, "Timing recovery for synchronous binary data transmission," Bell Syst. Tech. J., vol. 46, no. 3, pp. 593-622, 1967.

[7] R. A. Gibby and J. W. Smith, "Some extensions of Nyquist's telegraph theory," Bell Syst. Tech. J., vol. 44, no. 9, pp. 1487-1510, 1965. 